\title{
Effects of Maternal Dexamethasone Administration on Daily Foetal Movement Count and its Correlation with Doppler Studies and Non- Stress Test
}

\section{Original Article}

\author{
Youssef Mohamed Wahby, Ayman Nour Raslan, Hesham Mohamed El Ghazaly, \\ Abdallah Yehia El Kateb
}

Department of Obstetrics and Gynaecology, Kasr Al-Ainy Teaching Hospital, Cairo University

\begin{abstract}
Objective: To evaluate the effect of Dexamethasone on the daily foetal movement count and whether this affects haemodynamics.

Study Design: This is a cohort study involving pregnant women with a history of preterm labour, to follow up their daily foetal movement count for 5 days. Meanwhile, the non-stress test, middle cerebral and umbilical arteries Doppler indices were recorded before and after taking a designated Dexamethasone course. Each woman's data was considered as her control.

Patients and Methods: A total of 50 women with low risk, except a history of previous 1 or more preterm labour, were assigned to receive four separate injections of $6 \mathrm{mg}$ Dexamethasone, each given 12 hours apart. Doppler studies and cardiotocography traces were done before and after administration. Foetal movements were counted by mothers using Sadovsky method since the first dose and 5 days later. Paired T, McNemar's and Pearson's correlation coefficient tests were used.

Results: There were decrease in the MCA PI and RI (PI $p<0.001$, RI $p=0.002$ ), increase in foetal heart rate short term variability $(p=0.016)$ and decrease in the foetal movement count on Day $2(p<0.001)$.

Conclusion: Dexamethasone therapy affects the heart rate, haemodynamics and decreases daily foetal movement count.
\end{abstract}

Submitted: 30 November 2016, Accepted: 1 February 2017.

Key Words: Dexamethasone, Doppler, Mca, Ua, Non-Stress Test.

Corresponding Author: Youssef Mohamed Wahby, Mb/BCh, MSc., Tel.: 201003962650, E-mail: joewahby@doctor.com

ISSN: 2090-7265, February 2017, Vol. 7, No. 1

\section{INTRODUCTION}

Preterm birth, a leading cause of perinatal death and disability, is an important public health problem globally. Corticosteroid therapy, as proven by Liggins and Howie, could be used as a measure of decreasing foetal morbidities and mortalities. It was found to reduce the risks of complications of prematurity ${ }^{1,2}$.

Data from recent studies on prematurity bring into question about the side effects of corticosteroids on the influenced foetus. This was the basis of our study, as the decrease in foetal movements complaint has been recorded consistently by women receiving the Dexamethasone course.

There are many hypotheses to the mechanism of this effect. One is that corticosteroids exert a direct effect on the foetal brain: glucocorticoid receptors have been located throughout the foetal brain, especially in the hippocampus.
Another is that vascular tone after corticosteroid administration have shown discrepant results. While the prevailing effect is increased vascular tone, corticosteroids have potential biochemical actions for both vasoconstriction (e.g. reduced production of prostacyclin, inhibition of nitric oxide synthase) and vasodilatation (e.g. increased cyclic adenosine monophosphate responses) and may act by nongenomic mechanisms ${ }^{3-5}$.

Our work aimed at studying the effect of Dexamethasone on healthy foetuses, observing its effects on Doppler velocity waveforms, cardiotocography, and finally the influences on foetal movement.

\section{PATIENTS AND METHODS}

The study was conducted in the period between October 2012 and March 2013 at Kasr Al-Aini Obstetrics 
and Gynaecology Outpatient Clinic. Fifty participants, who came for ante-natal care, were recruited. We explained the purpose of the study and obtained a verbal consent from each. Proper clinical examination and full history taking were done to ensure the fitting of participants to criteria. A set of instructions regarding the method of drug use and other data collection procedures were given verbally and/or in the form of hand-outs.

Inclusion Criteria: Pregnant women with a history of preterm labour, gestational age between $32+0$ and $34+0$ and history of preterm labour.

Exclusion Criteria: Women who previously received a corticosteroid course, medical disorders, obstetric as well as foetal complications "e.g., congenital anomalies, intrauterine growth restriction IUGR", uterine anomalies that could alter Doppler findings "e.g., leiomyomata".

\section{Equipments \& Techniques:}

\section{Ultrasonography}

We used the Medison Sonoace model R7, with a C2 -8 probe, emitting $4.6 \mathrm{MHz}$ frequency and with a field of view of $68^{\circ}$.

Technique: Ultrasonographical and Doppler flow velocity waveforms studies were done with Pulse Wave Doppler after real-time colour flow localization of the UA (umbilical artery and the MCA (middle cerebral artery).

Three readings were taken for each waveform, and Resistivity Index (RI) and Pulsatility Index (PI) were recorded after the mean was calculated.

\section{Cardiotocography:}

The machine used was the Toitu MT-516 cardiotocogram. Cardiotocography strips were interpreted visually.

\section{Non-stress Test}

We used the parameters of normal non-stress test of the American College of Obstetricians and Gynaecologists, which entails the following:

Reactive: 2 or more accelerations in the span of 20 minutes, where acceleration is at least a $15 \mathrm{bpm}$ rise, occurring for at least 15 seconds.

Non-reactive: Baseline oscillation of less than $5 \mathrm{bpm}$ and/or absent accelerations. If less than 2 accelerations, non-stress test should be repeated for another 20 minutes to give time for sleep cycles ${ }^{6}$.

\section{Cardiotocography Study Parameters}

Other than the aforementioned criteria, a set of study parameters were followed up to give a more accurate perspective on foetal haemodynamics. These include: Foetal Heart Rate Baseline, Short Term Variability (STV), Long Term Variability (LTV), and Decelerations.

\section{Daily Foetal Movement count}

The Sadovsky method was employed. As it spans the whole day, participants were instructed to write down their findings in sheets designed for this purpose ${ }^{7}$. Drug Used:

The proprietary drug name is Epidrone, $2 \mathrm{ml}$ ampoule, consisting of $4 \mathrm{mg}$ of Dexamethasone Sodium Phosphate per $\mathrm{ml}$. The course was $3 / 4$ ampoules (6 mg) intramuscularly, every 12 hours, for 48 hours $(4 \text { doses })^{8}$.

\section{Study Procedure \& Data Collection Protocol:}

All participants were instructed about the framework of the research, which is as follows:

Day 0: UA and MCA Doppler values were measured; non-stress test was recorded at the clinic. Sadovsky method explained. Participants were instructed to start counting foetal movements as soon as they went home, to have the control section (designated Day C) of the foetal movement filled. A 1 st shot was to be taken at $10 \mathrm{pm}$ that day.

Day 1: Participants continue counting movement throughout the day. The $2^{\text {nd }}$ shot received at $10 \mathrm{am}$, and the $3 \mathrm{rd}$ shot received at $10 \mathrm{pm}$.

Day 2: Participants continue counting movement throughout the day. The 4 th shot received at $10 \mathrm{am}$.

Day 3: Participants come to the clinic at $12 \mathrm{pm}(60$ hours since the first dose). UA and MCA Doppler values measured, and non-stress test recorded.

Day 4, Day5: Participants continue counting movement throughout the day.

Day 6: Participants come to hand out the movement count sheet.

\section{Data Presentation and Statistical Methods:}

For this study, participants were their own control. For tests of significance, 0.05 was taken as the cut-off point for $\mathrm{p}$ value of significance. 


\section{Doppler Values:}

UA, MCA RI and PI values were expressed numerically in approximation to the second decimal. Paired $\mathrm{T}$ test was used to compare values prior to and after the course.

\section{Non-stress Test Values:}

The data in the strips were converted into 5 parameters; 1). The foetal baseline heart rate represented by its numerical value, 2) STV represented by 3 nominal values" Minimal: $<5 \mathrm{bpm}$, Moderate: 5- $25 \mathrm{bpm}$, Marked: $<25$ bpm, 3) LTV represented by the average number of the total long term cycles per recording. 4) Accelerations represented by their numerical value per recording. 5) Decelerations represented by their numerical value per recording.

The Paired $\mathrm{T}$ test was used for all parameters, except for the STV values. As a non-parametric test the McNemar's test was used for STV values?.

\section{Foetal Movement Count:}

The values of each of the three sittings were added to give an outcome of the counts per day, with one day before the start of the course as control. Since in each of the 3 sittings a participant had a maximum of 4 counts, the maximum value per day for this category was 12 . Paired $\mathrm{T}$ test was used to compare each day with the first control day. Correlation Tests:

Pearson's correlation coefficient was used to calculate correlations between all values ${ }^{10}$.

\section{RESULTS}

\section{Participants Demography:}

The study group had the following attributes summarized in the Table 1.

\section{Doppler Studies:}

Regarding the UA Doppler values, there were no significant changes. The mean RI difference was 0.013 . The mean PI was $\sim 0.97$ (1\%). As for the MCA Doppler values, there have been significant changes, especially in the PI, with means of $\sim 1.96$ before and $\sim 1.72$ after, and a mean difference of $0.23(12.2 \%)$, and a $p<0.001$. Consequently, there has been a significant change in the RI, with a mean difference of $0.02(2 \%)$ and a $p=0.002$. Results are shown in Table 2.

\section{Non-stress Test:}

The 5 variables of the NST (Non-stress Test) haven't showed much change. The baseline foetal heart rate showed a mean difference of only 0.46 of increase. The long-term variability cycles mean wasn't significance. Similarly, the number of accelerations showed a slight increase of, which was non-significant. Decelerations were not present at all before or after the course was given, so they were omitted from results and statistics (Table 3) The STV was the only variable which showed a significant change. . Before the course, all participants had moderate STV, but after the course, 7 (14\%) showed marked variability, which was considered significant by the McNemar's non-parametric test with a $p$ value of 0.016 (Fig. 1)

\section{Foetal movement:}

Apart from Day 2, which includes the $2^{\text {nd }}$ and the $3 \mathrm{rd}$ doses, there were no significant changes in the foetal movements. Day 2 had a mean of $11.1(5 \%)$, while the control for this category was 11.7 with a $p$ value $<0.001$. Day 4 narrowly escaped with a $p$ value of 0.064 and was deemed non-significant illustrated in Fig. 2.

\section{Correlation coefficients:}

Positive correlations were scarce even some of the expected correlations. The $r$ value and its significance between the variables with significant changes are listed in Table 4. A sole positive correlation was found between changes of Day 5 in foetal movements, and UA PI changes, with $r=0.282$ and $p=0.048$. Noteworthy, the latter change was statistically insignificant and its mean values before and after are almost the same.

However, the significantly decreased MCA RI had significant positive correlation with the Day 5 changes in foetal movements despite being non-significant, with $r=$ 0.281 and $p$ value $=0.048$. Another significant $r$ value was obtained between the relative changes in the STV and gestational age, which is a direct correlation. The $r$ value for this correlation is 0.448 , with $p$ value $=0.001$. 


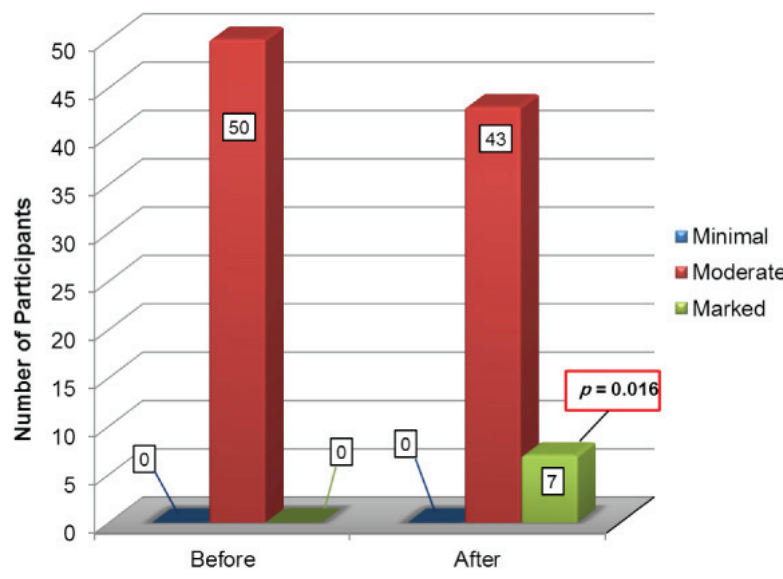

Fig. 1: Short term variability results.

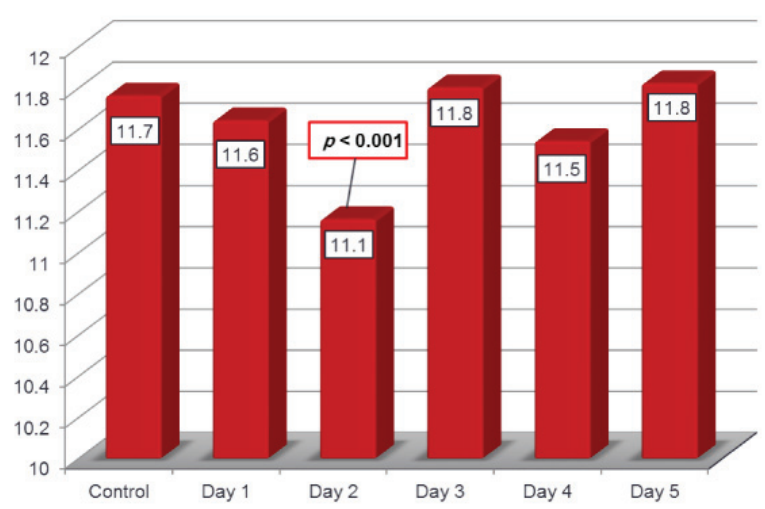

Fig. 2: Daily foetal movement count results.

Table 1: Descriptive data of patients.

\begin{tabular}{lccccc}
\hline & $\mathbf{N}$ & Min & Max & Median & Mean \\
\cline { 2 - 6 } Age & 50 & 20 & 38 & 26 & 26.6 \\
\hline Gestational age & 50 & $32^{+0}$ & $34^{+0}$ & $33^{+3}$ & $32^{+6}$
\end{tabular}

Table 2: UA and MCA Doppler Values.

\begin{tabular}{|c|c|c|c|c|c|}
\hline & Before & After & & & \\
\hline & Mean & Mean & Mean Difference & SD Difference & p value \\
\hline UA RI & $0.62 \pm 0.05$ & $0.63 \pm 0.05$ & -0.0134 & 0.05 & ns \\
\hline UA PI & $0.97 \pm 0.177$ & $0.97 \pm 0.15$ & -0.001 & 0.12 & ns \\
\hline MCA RI & $0.84 \pm 0.08$ & $0.82 \pm 0.08$ & 0.0208 & 0.04 & 0.002 \\
\hline MCA PI & $1.96 \pm 0.30$ & $1.72 \pm 0.29$ & 0.2316 & 0.21 & $<0.001$ \\
\hline
\end{tabular}

MCA: Middle cerebral artery UA: Umbilical artery PI: Pulsatility index RI: Resistivity index

Table 3: Baseline Heart Rate, Long term variability Mean, and Acceleration Values.

\begin{tabular}{|c|c|c|c|c|c|}
\hline & Before & After & & & \\
\hline & Mean & Mean & Mean difference & SD difference & $p$ value \\
\hline Baseline Rate & $138.8 \pm 7.92$ & $139.26 \pm 6.30$ & -0.46 & 4.32 & $0.45(n s)$ \\
\hline LTV Mean & $4.14 \pm 0.58$ & $4.14 \pm 0.61$ & -0.002 & 0.77 & $0.98(n s)$ \\
\hline Acceleration & $4.36 \pm 1.04$ & $4.30 \pm 1.07$ & 0.06 & 1.40 & $0.76(n s)$ \\
\hline
\end{tabular}

LTV: Long term variability 
Table 4: Correlation Coefficients \& Their Significance.

\begin{tabular}{lcc}
\hline & MCA PI & STV \\
\cline { 2 - 3 } STV & $\begin{array}{r}r=0.14 \\
p=0.31(n s)\end{array}$ & - \\
\hline \multirow{2}{*}{ Day 2 Foetal Movements } & $\begin{array}{r}r=-0.23 \\
p=0.97(n s)\end{array}$ & $\begin{array}{c}r=-0.007 \\
p=0.961(n s)\end{array}$ \\
\hline
\end{tabular}

STV: Short term variability

\section{DISCUSSION}

Our results are consistent with previous related research regarding the MCA PI, STV and foetal movements.

Noteworthy, we chose women who almost had no risks on their fetuses to avoid bias. A similar study by Chitrit et al. included 26 women whom were in risk. They used a dose of $4 \mathrm{mg}$ for 6 times and Doppler followup for 1 week, at 0 hour, 48 hours, 96 hours and 168 hours. They obtained a significant drop in the fetal middle cerebral artery pulsatility index (MCA PI) after 4 days of 0.28 with a $p$ value $<0.001$, but no significant changes were found in UA PI values. Subsequently, Chitrit et al. cite their hypothesis deemed it unclear and needs further animal studies ${ }^{11}$. In our study, we followed up foetuses between the 60 th and the $72^{\text {nd }}$ hour, but not on the $96^{\text {th }}$ hour, and still we have experienced this MCA PI drop, with a mean difference of 0.23 .

Similarly, Urban et al. recruited 67 women at risk of preterm labour to compare between Dexamethasone and Betamethasone. Thirty-four women received Dexamethasone, in the same dose as in our study. Follow up was done at 0,24 and 72 hours from first dose. They recorded a drop in the MCA PI values of 0.32 from the mean MCA PI after 72 hours. No significant change observed in the umbilical artery Doppler values. It is worth mentioning that 18 of the 34 women received tocolytics, and per their analysis, it didn't affect results ${ }^{12}$.

The sole study didn't record a significant decrease in the MCA PI was conducted on growth retarded foetuses $^{13}$, whereas Senat \& Ville proclaimed that the decrease continued for days. Per their analysis, such non-significant decrease may be attributed to the blood redistribution because of the growth retardation ${ }^{13}$.

Consistent with all previous studies, apart from Subtil et al, we found no significant changes in baseline foetal heart rate ${ }^{14}$.

We also recorded an increase in the baseline foetal heart rate, but it failed to produce a statistical significance.
Regarding the FHR variability, different results had been obtained. Senat et al and Mushkat et al., found no change in FHR variability. In contrast to all other work teams, Mushkat et al used the visual interpretation of CTG strips ${ }^{15,16}$.

Interestingly, Multon et al. who conducted their study on growth retarded foetuses only, alsofound no change with Dexamethasone ${ }^{17}$.

On the other hand, Dawes et al. concluded that dexamethasone administration normally causes a rise in foetal heart rate variation for up to a day. Magee et al. combined two steroids Dexamethasone and Betamethasone into one group to induce a significant increase of FHR STV. Interestingly, they attributed the increase in STV to the time of CTG recording. They mentioned Dawes et al. and Derks et al. studies for comparison. The patients described by Dawes et al. received steroids first thing in the morning and had traces repeated at the same time on subsequent days; short term variation was found to be increased on day one, as confirmed in this trial ${ }^{18-20}$.

In contrast, Derks et al. administered steroid at 18:00 $\mathrm{h}$ and then performed CTG traces between 10:00 $\mathrm{h}$ and 16:00 $\mathrm{h}$ on subsequent days; the short term variation was found to be significantly decreased by day two. Noteworthy, Derks et al used Betamethasone only ${ }^{20}$.

Mulder et al recorded an increase of FHR STV only in the Dexamethasone group, albeit with a later nonsignificant decrease, as the team was comparing both steroids. Comparing their study with Dawes et al and Derks et al., they concluded that an initial rise in foetal heart rate variation was observed on day 1 (significant for dexamethasone only), followed by a decrease in foetal heart rate variation on days 2 and 3 (significant for maternal betamethasone, but not for dexamethasone) ${ }^{19-21}$.

On the contrary, Rotmensch et $\mathrm{al}^{22}$ found a decrease in FHR STV 48 hours after first dose. They attributed the increased FHR variability in Dawes et al.'s study to the fact that $75 \%$ of the foetuses in their study were delivered by caesarean sections, thus increased FHR variability a consequence to foetal distress.

Similarly, the results by Subtil et al agreed with Mulder et al.'s analysis, whereas an increase at 8 hours, and a decrease on the $56^{\text {th }}$ and $80^{\text {th }}$ hours ${ }^{14,21}$.

Regarding FHR LTV, only Magee et al. recorded a significant decrease when combined the 2 groups. This may be explained by the repetition of the finding of decreased FHR LTV among studies of Betamethasone. 
Regarding accelerations, only Subtil et al and Rotmensch et al. recorded a change in the acceleration number. Both studies recorded significant decrease in the number of accelerations on the second day ${ }^{14-22}$.

In our study, we relied on visual interpretation that could only document strikingly significant CTG changes. Subsequently, no changes in FHR LTV, neither in accelerations were recorded, but there were 7 women with marked FHR STV had some effects.

These results coincide with previous studies that concluded increase in FHR STV, although our finding was around the $60^{\text {th }}$ hour; yet we didn't document an earlier change because of our study design. Thus, we documented the increase which marked a categorical difference (being defined as marked variability after being a moderate one).

Apart from studies by Rotmensch et al. and Magee et al., none recorded significant difference in foetal count. Magee et al. documented decrease in the first day relying on maternal perception during CTG recording, while Rotmensch et al. documented it around the second day relying on live ultrasonography-guided foetal movement count $^{18,22}$.

Our study design differed, since we relied on foetalkick counts all day not as a total continuous count, but in 3 groups with a limit of 4 counts per group as in the Sadovsky method. Despite being different, a significant decrease was recorded on the 2 nd day, consistent with Rotmensch et al. However, such decrease had no statistical correlation with any other parameters ${ }^{22}$.

Whether the maternal subjections towards foetal movements are factually true or erroneously reported, remains a matter of conjecture.

For the correlation coefficients, our research revealed a significant positive correlation between STV and gestational age. Alike, Lange et al observed that the more the gestational age increases, the more the sensitivity of the autonomic system, which helps in an increased response manifested in FHR variability.

Despite the non-significant decrease in foetal movements on Day 5, our results revealed a correlation between foetal movements ( 60 to 72 hours after last dose) and MCA RI, which may indicate a brain sparing effect. Conclusion

Dexamethasone administration resulted in some cardiovascular changes presented by increase in MCA blood flow and FHR short term variability. However, these changes were not strongly correlated with maternal perception of changes in foetal movements.
We didn't aim to determine whether Dexamethasone is better than other steroids for prevention of prematurity complications. Despite having no life threats, we recommend caution particularly with repeated Dexamethasone courses. The authors would like to certify that they have no affiliations with or involvement in any organization or entity with any financial interest (such as honoraria; educational grants; participation in speakers' bureaus; membership, employment, consultancies, stock ownership, or other equity interest; and expert testimony or patent-licensing arrangements), or non-financial interest (such as personal or professional relationships, affiliations, knowledge or beliefs) in the subject matter or materials discussed in this work.

\section{REFERENCES}

1. Saigal S, Doyle LW. An overview of mortality and sequelae of preterm birth from infancy to adulthood. Lancet 2007; 371(9608):261-269.

2. Liggins GC, Howie RN. A controlled trial of antepartum glucocorticoid treatment for prevention of the respiratory distress syndrome. Pediatrics 1972; 50(4): 515- 525.

3. McDonald TJ, Myers DA, Nathanielsz PW. Localization of type II glucocorticoid receptor (Gr) in the fetal sheep brain at 120 days of gestation (dGA). J Physiol 1993; 459: 331P.

4. de Kloet ER, Reul JMH, Sutanto W. Corticosteroids and the brain. J Steroid Biochem Molec Biol 1990; 37(3): 387- 394.

5. Walker BR, Williams BC. Corticosteroids and vascular tone: Mapping the messenger maze. Clin Sci (Lond) 1992; 82(6):597 -605.

6. American College of Obstetricians and Gynecologists: Antepartum fetal surveillance. Practice Bulletin No. 9, October 1999, Reaffirmed 2012.

7. Sadovsky E, Yaffe H. Daily fetal movement recording and fetal prognosis. Obstet Gynecol 1973; 41(6):845-850.

8. Antenatal Corticosteroids to Reduce Neonatal Morbidity and Mortality. RCOG Green-top Guideline No. 7. Royal College of Obstetricians and Gynecologists, London, UK, 2010.

9. Mc Nemar, Q. Note on the sampling error of the difference between correlated proportions or percentages. Psychometrika 1947; 12 (2): $153-157$. 
10. Karl Pearson. Notes on regression and inheritance in the case of two parents. Proceedings of the Royal Society of London 1895; 58: 240-242.

11. Chitrit Y, Caubel P, Herrero R, Schwinte AL, Guillaumin D, Boulanger MC. Effects of maternal dexamethasone administration on fetal Doppler flow velocity waveforms. BJOG 2000; 107(4):501- 507.

12. Urban R, Lemancewicz A, Przepieść J, Urban J, Kretowska M. Antenatal corticosteroid therapy: A comparative study of dexamethasone and betamethasone effects on fetal Doppler flow velocity waveforms. Eur J Obstet Gynecol Reprod Biol 2005; 120(2):170-174.

13. Senat MV, Ville Y. Effect of steroids on arterial Doppler in intrauterine growth retardation fetuses. Fetal Diagn Ther 2000; 15(1):36-40.

14. Subtil D, Tiberghien P, Devos P, Therby D, Leclerc $\mathrm{G}$, Vaast $\mathrm{P}$, et al. Immediate and delayed effects of antenatal corticosteroids on fetal heart rate: A randomized trial that compares betamethasone acetate, betamethasone phosphate and dexamethasone. Am J Obstet Gynecol 2003; 188(2): 524-531.

15. Senat MV, Minoui S, Multon $\mathrm{O}$, Fernandez $\mathrm{H}$, Frydman R, Ville Y. Effect of dexamethasone and betamethasone on fetal heart rate variability in preterm labour: A randomised study. $\mathrm{Br} \mathrm{J}$ Obstet Gynaecol 1998; 105(7):749-755.

16. Mushkat Y, Ascher-Landsberg J, Keidar R, Carmon E, Pauzner D, David MP. The effect of betamethasone versus dexamethasone on fetal biophysical parameters. Eur J Obstet Gynecol Reprod Biol 2001; 97(1):50-52.
17. Multon O, Senat MV, Minoui S, Hue MV, Frydman R, Ville Y. Effect of antenatal betamethasone and dexamethasone administration on fetal heart rate variability in growth-retarded fetuses. Fetal Diagn Ther 1997; 12(3):170-177.

18. Magee LA, Dawes GS, Moulden M, Redman CW. A randomised controlled comparison of betamethasone with dexamethasone: Effects on the antenatal fetal heart rate. Br J Obstet Gynaecol 1997; 104(11):1233-1238.

19. Dawes GS, Serra-Serra V, Moulden M, Redman CW. Dexamethasone and fetal heart rate variation. $\mathrm{Br} \mathrm{J}$ Obstet Gynaecol 1994; 101(8):675-679.

20. Derks JB, Mulder EJ, Visser GH. The effects of maternal betamethasone administration on the fetus. Br J Obstet Gynaecol 1995; 102(1):40-46.

21. Mulder EJ, Derks JB, Visser GH. Antenatal corticosteroid therapy and fetal behaviour: A randomised study of the effects of betamethasone and dexamethasone. Br J Obstet Gynaecol 1997; 104(11):1239-1247.

22. Rotmensch S, Lev S, Kovo M, Efrat Z, Zahavi $\mathrm{Z}$, Lev $\mathrm{N}$, et al. Effect of betamethasone administration on fetal heart rate tracing: A blinded longitudinal study. Fetal Diagn Ther 2005; 20(5):371-376. 